\title{
Brain Abscess in Pediatric Patients with Congenital Heart Disease: A Case Report and Review of the Literature
}

\section{Introduction}

Brain abscesses are frequent in pediatric patients with uncorrected or partially corrected cyanogenic congenital heart disease and there is a risk even in patients who are in the intervals between corrective surgeries. Next to brain abscesses resulting from complications of chronic otitis or sinusitis, brain abscesses secondary to heart disease are the most frequent etiology with an incidence reported of 5 to18 \% [1]. The pathophysiology is based on the right-to-left shunting present in cyanogenic cardiopathies that allows bacteria colonizing the airway to pass through the cerebral circulation [2]. Secondly, the polycythemia that these patients develop leads to tissue hypoxia and ischemia that together with the viscosity of the blood, creates a niche for the growth of bacteria [3].

Brain abscesses associated with congenital heart disease are typically caused by $S$. aureus and Streptococcus spp., whereas underlying dental and sinus infections are associated with polymicrobial infections, which often include Fusobacterium, Prevotella, Actinomyces, Bacteroides, and Haemophilus species. Polymicrobial abscesses account for up to $30 \%$ [3]. Regarding the clinical presentation, the most prevalent symptoms are fever $(90 \%)$, headache $(85 \%)$ and vomiting $(80 \%)$; seizures $(30 \%)$ are less frequent. The triad that includes: headache, fever and neurological symptoms is only present in $(15 \%)$ of the cases reported [3].

The treatment is controversial; however the use of thirdgeneration cephalosporins and metronidazole is recommended [4]. The level of evidence of steroid use in these patients is not yet clear because it is believed that they slow down the encapsulation process, they increase the risk of necrosis, reduce the penetration of the antibiotic to brain tissue and have a rebound effect. Medical treatment is recommended in patients without increased intracraneal pressure, with symptoms of less than 1 week long and abscess of less than $2 \mathrm{~cm}$ seen in tomography [5]. The advantages of surgery are that it allows cultures to be obtained and reduces the mass effect [5].

\section{Case Presentation}

We present the case of a 16-year-old male patient, born in Veracruz Mexico with history of pulmonary stenosis, hypoplastic right ventricle, interauricular communication and patent ductus arteriosus who had surgical intervention with Blalock Taussig shunt on November of 2003.

On July $11^{\text {th }} 2016$ he started with numbing of the left half of his face including the tongue, for which he didn't seek any medical attention. On July $18^{\text {th }} 2016$ he had a generalized seizure, which lasted 10 minutes, according to his family. On July 28th 2016 he started to lose strength of the left half of his body and wasn't able to walk or move his left arm. With these symptoms he was

\begin{tabular}{l} 
Case Report \\
Volume 11 Issue 1 - 2018 \\
Mercedes Macías Parra, Marte Hernández \\
Porras, Eduardo Arias de la Garza* and \\
Deborah Palacios Reyes \\
Department of Pediatric Infectious Disease, Instituto Nacional \\
de Pediatría, Mexico \\
*Corresponding author: Eduardo Arias de la Garza, \\
Department of Pediatric Infectious Disease, Instituto \\
Nacional de Pediatría, Mexico, Email: \\
lalo_arias@hotmail.com \\
Received: January 24, 2018 | Published: January 30, 2018 \\
\hline
\end{tabular}

admitted to our hospital where he also referred headache. CT scan showed hypodense images on right temporoparietal region and reinforcement images on right parietal region suggesting brain abscesses (Figure $1 \& 2$ ). With these findings, along with the clinical presentation, the diagnosis of brain abscess was made and antibiotic treatment was started with third generation cephalosporin, metronidazole and glucopeptide.

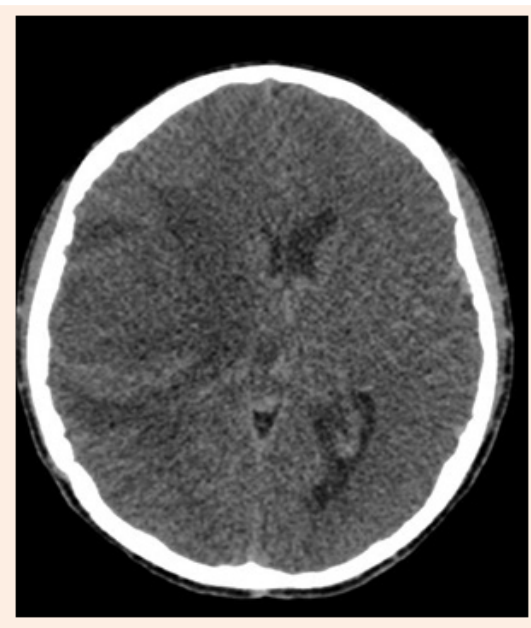

Figure 1

The patient was evaluated by the neurosurgery team who decided to differed surgical treatment because of the size of lesions in CT scan and improvement in next CT scan images. On August $8^{\text {th }} 2016$ a MRI (Figure 3) of the brain showed capsule formation. The patient continued with antibiotic treatment and dexamethasone in reduction dose. A new MRI of the brain, on August $29^{\text {th }} 2016$, showed abscesses of less than $3 \mathrm{~cm}$, this along with the patient improving neurological state and decide no need for surgical treatment. 


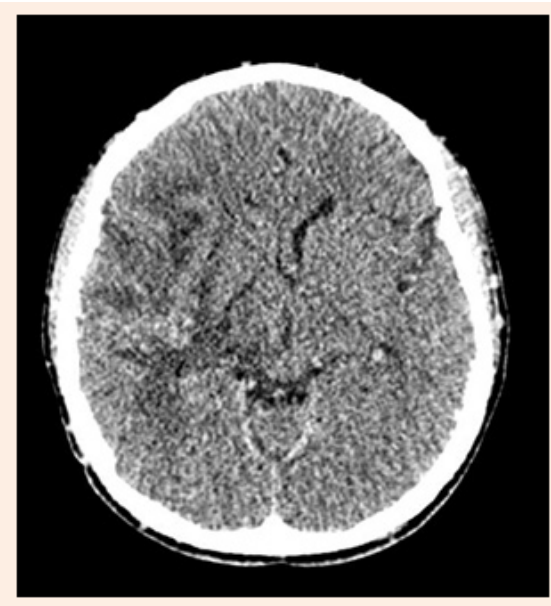

Figure 2

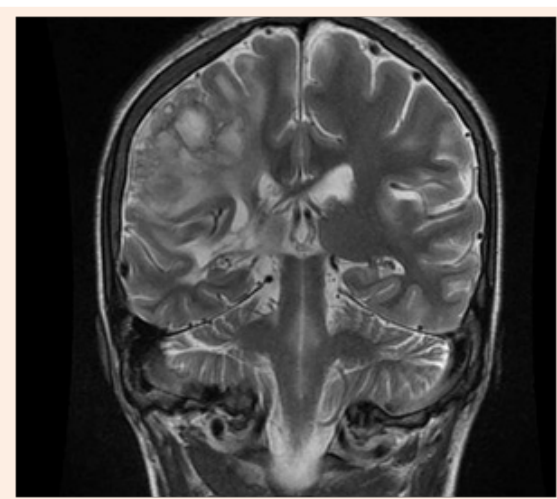

Figure 3

The patient completed 61 days of parenteral treatment with ceftriaxone, metronidazole and vancomycin. The last imaging study previous to the discharge of the patient showed brain abscesses of less than $0.5 \mathrm{~cm}$.

\section{Discussion}

As it was mentioned earlier, patients with palliate corrective heart surgery are at high risk of presenting brain abscesses. In most series the debutant age for cardiogenic brain abscess is the first decade of life with a mean age of 7.19 years but older ages are also reported [1].

In cardiogenic brain abscesses a hematogenous spread of bacteria can cause more than one brain lesion as it occurred the case report. The most common sites to find cardiogenic brain abscesses are frontal, temporal and parietal lobes followed by cerebellar and occipital lobes.

In most series, the most frequent symptoms are headache and vomiting, fever is not always present as it occurred in the case we present. The presence of focal neurological symptoms is also a frequent clinical feature and can indicate the location of the brain abscess. Seizures are reported with less frequency, some reviews report up to $25 \%$, the patient presented had one episode of generalized seizures.

The management of cardiogenic brain abscess can be more challenging compared to other abscesses because hypercoagulable states could complicate the surgery. Once it is established that no surgical procedure is needed for the treatment, as it happened in the presented case, antibiotic treatment should start with active coverage for the bacterial agents suspected and with adequate penetration to brain tissue. The suggested time of treatment is between 6 and 8 weeks with intravenously administered antibiotics. If the surgery is performed, antibiotic treatment can be shortened to 4 weeks.

For the follow up of these patients, imaging studies (CT scan or MRI) should be done every 2 weeks until the 6 th week of antibiotic treatment, after the surgical drainage or if the patient has neurologic deterioration [1]. For ambulatory follow up, it is recommended to do imaging studies 3 weeks after the antibiotic treatment is completed and every month until complete resolution is evident [1]. Acute phase proteins should also be measured periodically.

\section{References}

1. Udayakumaran S (2017) Forgotten? Not Yet. Cardiogenic Brain Abscess in Children: A Case Series-Based Review. World Neurosurg 107: 124-129.

2. Moorthy RK, Rajshekhar V (2008) Management of brain abscess: an overview. Neurosurg Focus 24(6): E3.

3. Tekkok IH, Erbengi A (1992) Management of brain abscess in children: review of 130 cases over a period of 21 years. Childs Nerv Syst 8(7): 411-416.

4. Sjolin J, Lilja A, Eriksson N, Arneborn P, Cars O (1993) Treatment of brain abscess with cefotaxime and metronidazole: prospective study on 15 consecutive patients. Clin Infect Dis 17(5): 857-863.

5. Raimondi AJ, Matsumoto S, Miller RA (1965) Brain abscess in children with congenital heart disease. I J Neurosurg 23(6): 588595. 